\title{
FOMENTO DEL EMPRENDIMIENTO EMPRESARIAL Y FORMACIÓN PROFESIONAL UNIVERSITARIA: CENTRO DE EMPRENDIMIENTO EN LA FCA, UNMSM
}

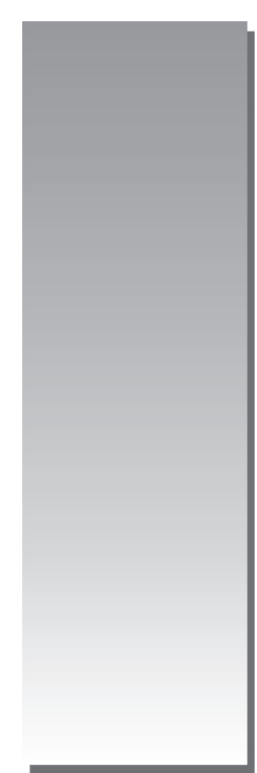

PROMOTION OF THE BUSINESS ENTREPRENEURSHIP AND UNIVERSITY PROFESSIONAL TRAINING: CENTER FOR ENTREPRENEURSHIP IN THE FACULTY OF ADMINISTRATIVE SCIENCES, UNMSM

\author{
Francisca Bouby Tolentino* \\ f_bouby@yahoo.com
}

\begin{abstract}
RESUMEN
El presente estudio tiene por objetivo destacar la necesidad de fomentar una actitud emprendedora en la formación profesional universitaria en un país en desarrollo como es Perú, con una estructura económica primaria exportadora, donde el empleo generado principalmente por las pymes muestra la necesidad de crear empleo con alto valor agregado. Por lo tanto, promover el emprendimiento a través de los futuros graduados es una de las nuevas y más relevantes funciones de la universidad peruana. Lo cual a su vez, pone en relevancia la urgente necesidad de una política educativa de Estado, que estimule el desarrollo educativo en todos los niveles, con competencias en investigación, innovación y emprendimiento.
\end{abstract}

\section{ABSTRACT}

This study highlights the need to promote an enterprising attitude on university professional training in an less developed country as Peru, with an primary exported economic structure where most of employment is explained by small and medium enterprises, which shows the imperative of an high aggregate value employment.

So, by promoting the undertaking amongst future graduates is considered one of the highest goals of the Peruvian university system, and this reveals the needing of an educational public policy that encourage educational development in all levels, with emphasis on research, innovation and enterprising attitude.

\footnotetext{
* Doctora en Economía por la UNAM, Magíster en Economía con mención en Política Económica, Docente principal a nivel Pre y Posgrado e investigadora en el Instituto de Investigación de la Facultad de Ciencias Administrativas (FCA), UNMSM. Ex Directora de la Escuela de Administración de Turismo de la FCA, UNMSM. Ex Vicerrectora Académica de la Universidad SISE. Consultora ESAN, GERENS.
} 


\section{INTRODUCCIÓN}

El presente estudio tiene por objetivo contribuir a destacar la necesidad de fomentar la toma de conciencia de una actitud emprendedora en la formación profesional universitaria.

En este sentido, se presenta el marco teórico, destacando que desde el siglo XVII existen aportes trascendentales sobre lo que significa ser emprendedor. Demostrando, asimismo, que la concentración económica va reduciendo el número de empresas, lo cual implica cada vez menos oportunidades laborales para los profesionales del área económico-empresarial, cuyo objetivo debe ser generar empresas para trabajar frente a la tendencia concentradora del poder económico.

Seguidamente se hace un abordaje sobre el fomento del emprendimiento empresarial en la formación profesional universitaria a nivel internacional. Destacando que es en Estados Unidos y Europa donde se ha marcado un liderazgo en la materia, a través de la creación de parques tecnológicos, incubadoras o viveros de empresas.

A continuación se aborda el fomento del emprendimiento en América Latina, donde se inició una corriente volcada hacia el fomento del emprendimiento empresarial y la formación profesional universitaria, desde finales de los noventa e inicios del presente siglo. Destacando que en Perú aún se carece de una política educativa de Estado que estimule el desarrollo educativo con competencias en investigación, innovación y emprendimiento.

Finalmente, se presenta una propuesta para estimular el emprendimiento empresarial en la formación profesional en la Facultad de Ciencias Administrativas (FCA), UNMSM, mediante la creación de un centro de emprendimiento, con la finalidad de contribuir a la formación de una cultura organizacional emprendedora.

\section{PLANTEAMIENTO DEL PROBLEMA}

La realidad de un país en desarrollo como Perú con una estructura económica primaria exportadora, donde las pymes son las principales fuentes generadoras de empleo, muestra la necesidad de generar empleo con mayor valor agregado. Por lo tanto, promover el emprendimiento a través de los futuros graduados es una de las nuevas y más relevantes funciones de la universidad peruana, asumida en nuestro país de modo muy incipiente, que contrasta con el avance en este tema a nivel de las universidades de América Latina y el mundo, lo cual pone en evidencia la carencia de una política educativa de Estado, orientada a fomentar la investigación, innovación y emprendimiento.

\section{OBJETIVOS}

\section{Objetivo general}

Contribuir a destacar la necesidad de fomentar la toma de conciencia de una actitud emprendedora en la formación profesional universitaria.

\section{Objetivos específicos}

Destacar la necesidad de convertir, frente al entorno económico-social cambiante, a la Facultad de Ciencias Administrativas, UNMSM, en paradigma de la formación profesional emprendedora a nivel universitario.

\section{JUSTIFICACIÓN}

Existe la necesidad de promover el inicio de un cambio de actitud frente a la tradicional salida laboral de los futuros graduados. Más que empleados, se debe formar emprendedores capaces de generar trabajo a través de la creación de empresas. Por lo tanto, es necesario estimular la vocación emprendedora de los estudiantes de la Facultad de Ciencias Administrativas, a través de la organización del Centro de Emprendimiento Empresarial en la facultad.

\section{HIPÓTESIS}

El fomento del emprendimiento empresarial está relacionado con la formación profesional universitaria.

\section{METAS ESPECÍFICAS}

- El presente trabajo será expuesto en eventos académicos para sustentar la necesidad de contribuir al desarrollo de una cultura organizacional orientada al emprendimiento como parte de la formación profesional universitaria.

- Demostrar la necesidad de la creación de un Centro de Emprendimiento Empresarial en la Facultad de Ciencias Administrativas. 


\section{MARCO TEÓRICO DEL TRABAJO}

Vivimos en una sociedad de organizaciones, en la cual hay una organización característica del sistema capitalista que es la empresa, cuyo principal protagonista es el empresario (Etzioni, 1987: 175). Según Schumpeter, el empresario es un maestro innovador, es "la fuerza detrás del desarrollo económico" (Schumpeter, 1934, citado en Etzioni 1987: 177), por lo que el concepto de empresario se asocia con el de emprendedor.

La creación de empresas constituye el centro de la investigación sobre emprendimiento (Veciana 1988 y otros, en Serarols-Tarré, 2006: 34), por lo que numerosos investigadores han intentado explicar el fenómeno de la creación de nuevas empresas desde diferentes perspectivas teóricas (Serarols-Tarré, 2006:34).

Desde el siglo XVII existen aportes trascendentales sobre lo que significa ser emprendedor.

Según la teoría clásica (Adam Smith, David Ricardo, etc.), el empresario del siglo XVII era quien aportaba el capital a su empresa, lo administraba y controlaba. Marx mantiene el concepto de empresario capitalista en sus aportes, según los cuales, el capitalista aporta capital y obtiene beneficio como apropiación de plusvalía del trabajador (Veciana, 1988:3-4, en Delicio, 2006: 45)

Según López Vidal, es Cantillón quien utiliza por primera vez el término "entrepreneur" en su obra Essai sur la nature du commerce, en general, y lo define como "el agente que compra medios de producción a ciertos precios, para combinarlos en un producto que venderá a un precio incierto en el momento del compromiso de sus costes" (López Vidal, 1997:30, en Delicio 2006: 45). Destacándose dos elementos importantes del ser emprendedor. De un lado, cambiar y transformar recursos a fin de satisfacer necesidades y, del otro, capacidad de asumir riesgos, característica básica del emprendedor.

Jean Baptiste Say, a principios del siglo XIX, destaca la organización y la coordinación de los factores productivos, y, siguiendo a Cantillon, señala que "entrepreneur es una persona que cambia recursos económicos desde zonas de baja productividad y rendimientos a zonas de alta productividad y mayor rendimiento" (López Vidal, 1997:30).

La empresa tiene como característica inherente la innovación y una de las características del empresario emprendedor es ser innovador; es quien realiza las modificaciones tecnológicas necesarias para que se genere el cambio. En síntesis, empresario es quien realiza innovaciones, es decir, quien concreta las acciones. Peter Drucker remarca este concepto al decir: "Lo que define al entrepreneur es que busca el cambio, responde a él y lo explota como una oportunidad" (Drucker, 1995:380, en Delicio 2006: 46).

De otro lado, aún está en debate si el emprendedor nace o se hace. Esto da lugar a dos enfoques: un enfoque psicológico, que se basa en los rasgos personales, y sostiene que el emprendedor nace; el otro enfoque es el sociológico, que sustentado en la conducta del emprendedor, establece que el emprendedor se hace.

Para Drucker, entrepreneurship es una disciplina que se puede enseñar y aprender y, por lo tanto, aplicar. Es consecuencia de un trabajo duro y sistemático, y como en toda actividad profesional, el emprendedor necesita formación y capacitación.

El ser humano ha sido el artífice de todas las innovaciones y modificaciones de su entorno, buscando mejores condiciones de vida, utilizando tres elementos básicos en su accionar, tales son: noción de cambio, noción de acción y noción de mejoramiento (Varela 2008: 8). El espíritu humano es innovador, generador de emprendimientos, necesarios para la creación de empresas. El proyecto Global Entrepreneurship Monitor (GEM) reconoce el papel fundamental que tiene la creación de empresas para el crecimiento económico de los países (Valera Villegas, 2005:75, en Delicio 2006: 46).

En Latinoamérica en general, se afirma que el sistema educativo, aún con limitaciones, tiene un papel importante en el desarrollo del proceso emprendedor; destacándose el aporte de la universidad en la adquisición del conocimiento técnico específico. Se reconoce, asimismo, que la actividad emprendedora es consecuencia también de la experiencia laboral previa de los individuos (Kantis et al, 2002:40-41, citado en Delicio 2006:57).

Por otro lado, existe un debate entre formar gerentes o empresarios, en las carreras que se dictan en las facultades de ciencias económicoempresariales, las cuales poseen planes de estudios adaptados al ejercicio profesional en sus respectivas disciplinas. 
Sin embargo, la realidad del mercado laboral es que este se encuentra cada vez más restringido, tanto para el profesional independiente como para el que se halla en relación de dependencia. La concentración económica (“... en los últimos decenios del siglo pasado... el proceso de concentración se presentó bajo un doble aspecto de control progresivo - por una empresa de un mercado determinado - de un producto o una clase de productos y de articulación bajo un solo grupo económico de las distintas fases de un proceso productivo, desde la obtención de una materia prima hasta el suministro de un producto final", en Furtado Celso, (1968), La concentración del poder económico en Estados Unidos y sus proyecciones en América Latina, Estudios Internacionales) va limitando el número de empresas, siendo éste cada vez menor, ofreciéndose cada vez menos oportunidades laborales para los profesionales del área económico-empresarial; cuyo objetivo debe ser generar empresas para trabajar frente a la tendencia concentradora del poder económico.

Es práctica común que los planes de estudios de las escuelas profesionales del área económicoempresarial, especialmente las de administración, estén orientados a formar directivos (gerentes) y no a la formación de emprendedores. Existen características específicas en la formación de un directivo de empresas que muestran diferencias marcadas con la formación de un emprendedor. Así, el gerente actúa en función de objetivos establecidos por otros; en cambio, el emprendedor fija sus propios objetivos en su empresa. En el emprendedor predomina la creatividad y la innovación; de su espíritu empresarial, esfuerzo y visión del contexto, surgen nuevas ideas para nuevos emprendimientos (ver Varela, 2008).

El emprendedor debe desarrollar, como parte de su formación profesional, creatividad, capacidad de riesgo y conocimientos para estar atento a los cambios del entorno, comprenderlos y definir la idea de negocio.

El gerente debe dominar sus funciones orientadas a una eficiente coordinación de los factores productivos, generando motivación, estableciendo incentivos, entendiendo la cultura organizacional para un adecuado manejo con individuos y grupos, aplicando medidas de control adecuadas a la problemática de la empresa.
En síntesis, tanto management como entrepreneurship son disciplinas objeto de enseñanzaaprendizaje en la universidad, cada una tiene sus propias especificidades, pero también hay puntos en común. Siendo el management una disciplina que se enseña tradicionalmente en la universidad, en cambio, el entrepreneurship se viene incorporando paulatinamente como una disciplina nueva en los claustros universitarios.

\section{MÉTODO}

El presente estudio es de tipo descriptivo, correlacional. En el diseño de la investigación se ha empleado el análisis de corte transversal. Las fuentes de información son básicamente secundarias. Se ha empleado el método analítico-sintético, y una combinación de métodos de investigación cualitativos y cuantitativos.

\section{RESULTADOS}

En relación con el fomento del emprendimiento empresarial en la formación profesional universitaria a nivel internacional, se llegó a los siguientes resultados:

En España, La Universitat Politécnica de Catalunya (UPC) cuenta con una serie de contactos y convenios con organismos públicos y empresas privadas, a fin de fomentar la actividad emprendedora entre los estudiantes. Entre sus iniciativas destacan: los convenios de cooperación educativa con empresas para estimular la actividad emprendedora entre los estudiantes de los últimos semestres académicos. El proyecto de fin de carrera, cuyo desarrollo y defensa es obligatorio para obtener el título profesional. Las junior empresas, que son asociaciones sin fines de lucro, formadas y gestionadas por estudiantes.

La Universidad Autónoma de Madrid cuenta con el Centro de Iniciativas Emprendedoras, para el fomento de la cultura emprendedora y el autoempleo. Entre sus actividades, se destacan: las Jornadas de Sensibilización, cuyo objetivo es crear conciencia y lograr un cambio cultural hacia el autoempleo. Capacitación, ofrecida a los emprendedores con el propósito de complementar sus conocimientos técnicos, de tal forma que se adecúe lo máximo posible a las necesidades derivadas de su idea de negocio. Asesoría en la elaboración 
de proyectos empresariales, a fin de brindar a los emprendedores las herramientas para dar forma a su idea de negocio y desarrollarlo hasta su puesta en marcha.

En México, el Instituto Tecnológico y Estudios Superiores de Monterrey (ITESM) ha creado el Programa Emprendedor Universitario a fin de desarrollar el potencial emprendedor entre sus estudiantes. Su implementación se realiza a través de las siguientes áreas: Área motivacional, cuya tarea es la difusión de logros alcanzados, asimismo, la organización de eventos con emprendedores y la organización de muestras empresariales; el Área Académica, que lleva a cabo el proceso de enseñanza-aprendizaje, proporcionando un sustento conceptual a las actividades del programa por medio de una serie de iniciativas, entre las que destacan: la capacitación a profesores en la tarea de identificar a los estudiantes emprendedores; el Curso Sello, como parte del plan de estudios de cada carrera, siendo su objetivo “... que el alumno fortalezca su potencial emprendedor a través del desarrollo de sus habilidades para definir, planear y poner en marcha un proyecto innovador". (Sánchez Lozano, 1998: 161) y el diplomado en Desarrollo de Micro y Pequeña Empresa. El Área de Apoyo Institucional, cuyo fin es brindar apoyo institucional a través de recursos humanos y materiales a los estudiantes que culminan su proyecto en una MYPE.

En Costa Rica, el Instituto Tecnológico de Costa Rica (ITCR) tiene por finalidad llevar a cabo un proceso de desarrollo y difusión de la filosofía emprendedora y del espíritu empresarial entre sus estudiantes y docentes, a partir del Programa de Emprendedores creado en 1995. Este programa busca generar un cambio a la tradicional salida laboral de los futuros graduados, de empleados a emprendedores capaces de generar trabajo a través de la creación de empresas.

En Chile, La Universidad de Concepción de Chile, desde inicios del presente siglo, puso en marcha un Plan de Fomento de la Vocación Emprendedora entre sus estudiantes, a través del Centro de Incubación de Empresas. Complementando esta iniciativa, con el inicio, en el año 2002, de un Programa de Formación de Profesionales Emprendedores en la Universidad de Concepción, cuyo fin es generar competencias emprendedoras en la formación de profesionales, mediante nuevos contenidos y metodologías de enseñanza - aprendizaje. Posteriormente se dio inicio al Programa Idea-Incuba, orientado a la incubación de empresas de alta tecnología, dedicada a estudiantes y profesores, pero también abierta a la comunidad.

En Brasil, la Universidad Estadual de Campinas (UNICAMP) ha establecido lazos con el sector productivo brasileño, su presupuesto proviene de los recursos públicos del Gobierno del estado de San Pablo. Con este fin, en Brasil existe la Financiadora de Estudios y Proyectos (FINEP), que se encarga de gerenciar el Fondo Nacional para el Desarrollo Científico y Tecnológico.

A fin de aprovechar los fondos e incentivos fiscales existentes en Brasil, la UNICAMP ha creado en 1994 el Centro de Incentivo a la Asociación Empresarial.

La Universidad de Sao Paulo (USP) ha desarrollado un Programa de Incubadora de Empresas, cuya finalidad es estimular el surgimiento de empresas y unidades de negocios a partir de proyectos generados al interior de la universidad. (Azevedo Fonseca, 1998:263). Este proyecto se enfoca como un proyecto dedicado a la prestación de servicios de la universidad.

En Argentina, la Universidad de San Andrés (USA), ha sido pionera en el desarrollo del estímulo emprendedor universitario. Ha creado la Cátedra Karel Steuer, cuyo objetivo es impulsar la formación de los estudiantes con actitud emprendedora, con capacidad de identificar oportunidades, desarrollar nuevos proyectos, asumir riesgos, asimismo, adquirir un compromiso ético con la sociedad (http://www.udesa.edu.ar/entrepreneurship). Esta universidad ha incentivado la formación emprendedora como parte de los contenidos de sus planes de estudios. A través del Programa Entrepreneurship, instrumenta una serie de actividades relacionadas con la investigación, capacitación y sensibilización sobre la problemática del emprendedor (http://www.udesa. edu.ar/Propuesta-San-Andres/eEntrepreneurship/ La-Organizacion/Mision).

En Colombia, es donde más se ha contribuido al desarrollo de la educación empresarial. Un ejemplo es la creación del Centro de Desarrollo del Espíritu Empresarial (CDEE) de la Universidad Icesi. Otra experiencia importante es el Programa de Emprendedores de la Universidad Javeriana de Cali. La Universidad de los Andes, en Colombia, cuenta con el Centro de Iniciativa de Emprendimientos Sociales, responsable de formular proyectos 
de investigación y de impartir cátedras en los diferentes programas de pregrado, maestría y educación continua. Un aspecto destacable de este centro educativo es su participación en la Red de Conocimientos sobre Emprendimientos Sociales. (Borjas, 2012).

Según Terapuez, en Colombia, al incluirse el tema del emprendimiento en la agenda de gobierno del presidente Uribe, en el año 2002, se logra generar la dinámica necesaria para involucrar a entidades públicas y privadas, de distintos niveles, para operar de forma coordinada en dicho tema, bajo un esquema de política pública (Terapuez, 2013: 275).

Cabe destacar que en la formulación de dicha política de emprendimiento se tomaron en cuenta tres componentes: "las instituciones, la base legal y las intervenciones que los agentes públicos y privados realizan mediante planes, programas y proyectos en las distintas fases de la cadena de valor de la creación de empresas" (Terapuez 2013: 280). Siendo el objetivo de las acciones de la política de emprendimiento: los emprendedores, posibles emprendedores y sus empresas.

En Venezuela, cada universidad desarrolla su propio modelo de educación empresarial, (Borjas 2012). La Universidad Monte Ávila (UMA) tiene un modelo de enseñanza que combina la autogestión con el emprendimiento social; el proceso de educación empresarial está inmerso en el eje transversal de las carreras de pregrado y posgrado, y su objetivo es desarrollar las competencias que el mercado venezolano requiere. La Universidad Central de Venezuela (UCV), pionera en el desarrollo del emprendimiento, ha desarrollado el Observatorio de la Pyme, como una forma de reconocer el impacto que la pequeña y mediana empresa tiene en los procesos de ajustes de la economía debido a su papel protagónico en la creación de empleo, producción e innovación.

La educación empresarial en la Universidad Simón Bolívar (USB) pone énfasis en los parques tecnológicos como una forma de apostar al desarrollo de las empresas a través del proceso de incubación. La USB, a través del Parque Tecnológico Sartenejas, ha creado una incubadora de empresas diseñada para apoyar la creación de pymes y generar nuevas actividades en las empresas existentes mediante soporte estratégico y operacional.

\section{DISCUSIÓN}

El espíritu empresarial es considerado como pilar fundamental para el desarrollo económico en los países, por lo cual se requieren políticas que orientan esta actividad, a fin de fomentar la formación en competencias del emprendimiento en los procesos educativos (ODCE 2003, 2008 y Conferencia de las Naciones Unidas, 2011, citado en Borjas, 2012).

El emprendimiento es complejo, tiene a su vez factores que lo conforman, tales como el desarrollo de las competencias del estudiante, la tecnología en los procesos de innovación y creatividad, los recursos materiales y financieros y los conocimientos de esta disciplina. (Nonaka y Takeuchi, 1993, citado en Borjas, 2012). El contexto cultural es, para Kantiss (2011), un factor que puede determinar el desarrollo de las conductas emprendedoras, la cultura organizacional (Borjas, $2010,2011)$ y el valor agregado que puede generar la acción conjunta de tres: Estado, educación y empresa.

\section{Emprendimiento empresarial y formación profesional en la universidad: abordaje global}

La literatura existente sobre educación empresarial se refiere, desde un primer enfoque, a si el emprendimiento se puede enseñar o no (Henry, Hill y Leitch, 2003), mientras que otros enfoques están orientados a determinar cuál es la mejor manera de hacerlo (Kuratko, 2004).

Desde finales del siglo XX, autores citados por Borjas (2012), tales como Gibbons, Limoges, Newton, Schwartzman, Scott y Throw (1994) y Slaughter y Leslie (1997), empezaron a establecer los fundamentos teóricos de la educación empresarial, que respalda una visión complementaria de la actividad tradicional, denominada la tercera misión de la universidad (Bueno y Casani, 2007), para describir las actividades que las universidades llevan a cabo con los diferentes agentes sociales con que se relacionan y a los que transfieren su conocimiento fuera del ámbito académico (Nonaka y Takeucchi, 1993).

Gibb A. (1993) sostiene que la educación empresarial debería tener presencia en las currículas de todas las escuelas y facultades.

Para Blenker (2006), el quehacer de las universidades debe desarrollarse a través de tres ejes: el 
saber, la universidad emprendedora y el comportamiento emprendedor. Este modelo está fundamentado en la teoría de la triple hélice (Etzkowitz y Leydesdorff, 2000), referido a la interacción entre las universidades, el sector empresarial y el sector público, que aportan, respectivamente, el conocimiento y el desarrollo de las competencias personales y profesionales del estudiante.

Stokes y Wilson (2010) sostienen que la educación empresarial en la universidad, tiene tres dimensiones: contexto, procesos y conductas. El contexto está conformado por sectores de mercado, factores sociales y ambientales; el proceso considera cómo se desarrolla un nuevo emprendimiento o servicio; y las conductas definen las destrezas, las características y las actitudes del emprendedor.

La educación empresarial, para Varela y Bedoya, está orientada a "la formación de líderes empresariales capaces de crear organizaciones, sean ellas privadas o públicas, con ánimo de lucro o sin él, en cualquier sector de la economía, con cualquier estructura jurídica, con cualquier objetivo, de cualquier tamaño, con cualquier estructura administrativa” (Varela y Bedoya, 2006: 23).

\section{Emprendimiento empresarial y formación profesional en la universidad: abordaje en Latinoamérica}

Según Borjas (2012), el desarrollo de la educación empresarial emprendedora en Latinoamérica se ha hecho cada vez más evidente con las actividades que desarrollan las universidades en el contexto latinoamericano. Así, en el marco del Programa Emprendedores de la Facultad de Contaduría y Administración (FCA) de la Universidad Nacional Autónoma de México (UNAM), los estudiantes de pregrado tienen la posibilidad de consolidar sus proyectos empresariales a través de materias presentes en la currícula de estudios.

En Brasil el Estado, el sector educativo y el sector privado han establecido como factor prioritario el fomento de la capacidad emprendedora en la educación.

En Colombia, Ecuador, Perú y Venezuela, los programas de emprendimiento han comenzado a finales de la década de los noventa y a comienzos de este siglo (Lozano, 2004: 120).

En Perú se viene incentivando la actividad emprendedora con programas como Mi Empresa, iniciativa del Ministerio de Trabajo y Promoción de Empleo. En cuanto a la formación emprendedora en las universidades, ha venido aumentando de cierta manera el incentivo para la creación de empresas, aunque a un nivel incipiente (GEM 2008: 55).

En Ecuador, la Universidad Andina Simón Bolívar ha desarrollado un Observatorio de Pymes a fin de contribuir al fortalecimiento de este sector.

En Venezuela, Pietrosemolli (2006) argumenta que no existe un marco sólido de referencia que permita el surgimiento y el mantenimiento de un espíritu empresarial que sustente el crecimiento y el desarrollo nacional. Sin embargo, esta situación parece haber cambiado en los últimos años (Borjas, 2012).

Propuesta para estimular el emprendimiento empresarial en la formación profesional en la Facultad de Ciencias Administrativas (FCA), UNMSM

Se considera que es necesario difundir, entusiasmar, formar y ayudar al nacimiento de iniciativas emprendedoras entre los estudiantes, desde su ingreso a la universidad. Desde allí se puede generar un modelo de promoción de empresarios innovadores, basado en el desarrollo de competencias personales y competencias de conocimiento y teniendo como objetivo generar un empresario integrado al contexto empresarial.

Con base en la experiencia de universidades latinoamericanas y a nivel global, abordadas en este trabajo, y tomando en cuenta las características de las escuelas académico-profesionales de la Facultad de la Ciencias Administrativas, se plantea la creación de un Modelo de Emprendimiento de la Facultad de Ciencias Administrativas, con la finalidad de contribuir a la formación de una cultura organizacional emprendedora.

\section{Modelo de Emprendimiento de la Facultad de Ciencias Administrativas (Enfoque integral)}

Objetivos: Facultad de Ciencias Administrativas de la UNMSM, líder en emprendimiento empresarial universitario.

Iniciar un programa de emprendimiento, instrumentando una serie de actividades relacionadas con la investigación, capacitación y sensibilización sobre la problemática emprendedora. 
Áreas de desarrollo: El Centro de Emprendimiento de la FCA deberá tener cuatro áreas: Área Académica, Área de Formación y Capacitación, Área de Fomento y Difusión y Área de Estimulación.

Área Académica: Se orientará a capacitar en materia de emprendimiento a los estudiantes como parte de su formación académica, desde el ingreso hasta su egreso de la FCA, tanto a nivel de conocimientos teóricos como actividades prácticas.

Área de Formación y Capacitación: Para un adecuado fomento de la actividad emprendedora en la FCA, será indispensable una capacitación adecuada de su plana docente por docentes expertos de universidades extranjeras, con trayectoria en emprendimiento universitario. Para lo cual se deberá equipar tanto la biblioteca como el centro de documentación con material bibliográfico adecuado para la capacitación y formación de docentes y estudiantes.

Área de Fomento y Difusión: Su objetivo será difundir la labor emprendedora entre los estudiantes, mediante actividades, tales como encuentro de emprendedores, congresos y eventos sobre temática emprendedora. Una página web para difundir la información de interés para emprendedores, así como foros de discusión de la temática, donde docentes y estudiantes puedan encontrarse e intercambiar opiniones sobre emprendimiento.

Área de Estimulación: Orientada a fomentar la participación de los estudiantes de la FCA en ferias y concursos, con proyectos innovadores ge- nerados por ellos y premiación de los mismos. Se deberá fomentará el apoyo a emprendedores no universitarios, mediante el asesoramiento y cursos de capacitación brindados por docentes de la FCA con la colaboración de estudiantes. De este modo, la FCA no solo realizará una importante labor de extensión universitaria, sino también se logrará una experiencia valiosa para los estudiantes en su futura acción emprendedora.

Mecánica de Funcionamiento: Las actividades emprendedoras de la FCA se desarrollarán en dos etapas:

$1^{\text {a }}$ Etapa: Se pondrá en funcionamiento la Escuela de Emprendedores, por un periodo de dos años. Comprende el fomento y el apoyo a emprendedores.

$2^{\mathrm{a}}$ Etapa: Se pondrá en funcionamiento el Centro de Emprendimiento. Comprende el apoyo a la creación y al desarrollo de empresas.

Este modelo tiene un enfoque integral, ya que ambas etapas se retroalimentan permanentemente; siendo la primera fase, el Fomento a Emprendedores, de suma importancia para el correcto funcionamiento del modelo. Por ello este enfoque enfatiza la necesidad de fomentar actividades de sensibilización y dinamización del emprendimiento. Contrariamente, muchos modelos hallados en la literatura revisada en este estudio no tratan o no enfatizan este tema, probablemente porque han supuesto que dentro de sus contextos existe una adecuada cultura emprendedora, suficiente para fomentar el emprendimiento.

\section{MODELO DE EMPRENDIMIENTO DE LA FCA (ENFOQUE INTEGRAL)}

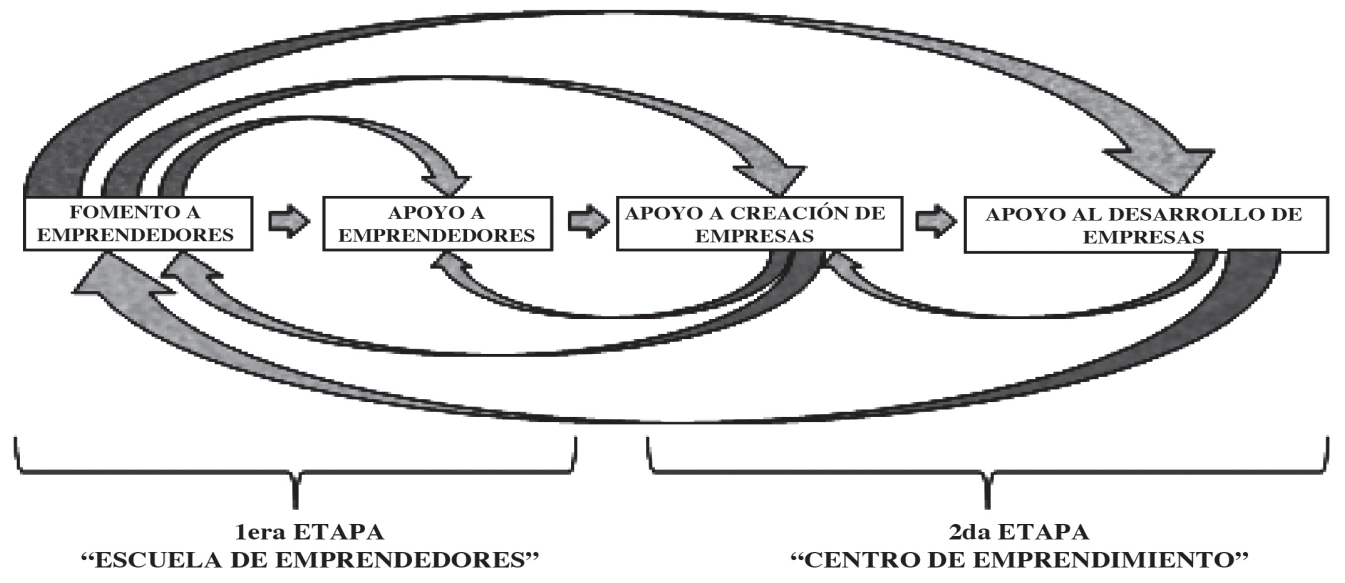




\section{CONCLUSIONES}

1. Desde finales del siglo XX, varios autores empezaron a establecer los fundamentos teóricos de la educación empresarial emprendedora, que respalda una visión complementaria de la actividad tradicional, denominada la tercera misión de la universidad.

2. El desarrollo de la educación empresarial emprendedora en Latinoamérica se ha hecho cada vez más evidente con las actividades que desarrollan las universidades.

3. El fomento de la educación emprendedora es un campo relativamente nuevo para la mayoría de las universidades de nuestro país.

4. Es necesario propiciar el debate sobre la necesidad de implementar una política educativa de Estado que estimule la incorporación de programas académicos en educación empresarial en las instituciones educativas superiores que tengan como propósito el desarrollo educativo con competencias en investigación, innovación y emprendimiento.

5. Desde la universidad se puede generar un modelo de promoción de empresarios innovadores, basado en el desarrollo de competencias personales y competencias de conocimiento y teniendo como objetivo generar un empresario emprendedor integrado al contexto empresarial.

6. Se plantea la necesidad de la creación de un Centro de Emprendimiento en la Facultad de Ciencias Administrativas, con la finalidad de contribuir a la formación de una cultura organizacional emprendedora.

\section{REFERENCIAS BIBLIOGRÁFICAS}

Borjas de Xena, Leslie. (2012). La educación empresarial en instituciones de educación superior venezolanas. En: Rev. Estudios Gerenciales Vol. 28, n 125 oct-dic 2012.

Chicoma, Jose Luis. (2011). El desafío de los emprendedores, Ministerio de Educación, Congreso Internacional Competencias y Capacidades, marzo 2011, Lima, Perú.

Delicio Fabián Andrés. (2006). Estrategias para el fomento del emprendedurismo universitario en la Facultad de Ciencias Económicas y Sociales de la Universidad Nacional de Mar del Plata. En: Rev. Faces, año 12, № 26, mayo/agosto 2006, Mar del Plata, Argentina.
Durand, Francisco. (2010).El emprendedurismo: más allá de la ideología. DESCO. En: Rev. QUEHACER, enero 2010.

DOING BUSINESS 2011. (2010). Creando oportunidades para los emprendedores, BIRF-BM http:// espanol.doingbusiness.org/ /media/FPDKM/ Doing\%20Business/Documents/Annual-Reports/Overview/DB11-Overview-Spanish.pdf

Global Entrepreneurship Monitor GEM. (2009). Empreendedorismo no Brasil, Instituto Brasileiro da cualidade e productividade, Curitiba, Brasil. http://www.gemconsortium.org/docs/download/449

Jiménez-Sáez, Fernando y Arroyo-Vázquez, Mónica. (2007). El Fomento del Emprendedurismo Universitario a través de un Modelo Integrador. Instituto Ingenio (CSIC-UPV), España. http://digital.csic.es/ bitstream/10261/20580/1/AC265_1_E-archivos-185.pdf

Naciones Unidas. (2007). Informe de la reunión de expertos sobre el aumento de la participación de las pymes de los países en desarrollo en las cadenas de valor mundiales, Ginebra. http://unctad.org/es/Docs/ c3em31d3_sp.pdf

Paniago, Robson (2011) O que é empreendedorismo. http://www.administradores.com. br/informe-se/administracao-e-negocios/oque-e-empreendedorismo/42396/

Reinoso Lastra, Juan Fernando. (2012). Aplicación de un ambiente virtual de aprendizaje orientado a la formación empresarial. En: Rev. Estudios Gerenciales, Vol. 28, № 122, enero-marzo 2012.

Revuelto T., Lorenzo y Fernández G., Rafael. (2009). Análisis y resultados de un modelo de evaluación, apoyo y mejora de proyectos empresariales impulsados por jóvenes emprendedores. En: Rev. Estudios Gerenciales, Vol. 25 NN $^{\circ}$ 113, octubre-diciembre 2009.

Tarapuez Ch. E·., Osorio C. H. y Botero V. J. (2013). Política de emprendimiento en Colombia, 2002-2010. En: Rev. Estudios Gerenciales Vol. 29, N 127, abril-julio 2013.

Uribe Toril, Juan y Valenciano, Jaime de Pablo. (2011). Revisando el Emprendedurismo. En: Boletín Económico de Ice $\mathrm{N}^{0} 3021$, diciembre 2011. 